19951,9510

N95- 25930

44645

P. 8

\title{
APPENDIX $\mathbf{R}$
}

\section{Cosmic Ray LET spectra and Doses on Board the Cosmos-2044 Biosatelitte}

by

V.E. Dudkin, E.E. Kovalev, Yu.V. Potapov, B.V. Benton, A.I. Frank E.R. Benton, J.W. Watts, Jr., T.A. Parnell, B. Bchopper,

B. Baican, G. Reitz. H. Bücker, R. Facius,

R. Beaujean and $C$. Heilmann 


\title{
COSMIC RAY LET SPECTRA AND DOSES ON BOARD COSMOS-2044 BIOSATELLITE
}

\author{
V. E. Dudkin, E. E. Kovaley, Yu. V. Potapov, E. V. Benton, † A. L. Frank, †. R. Benton, $†$

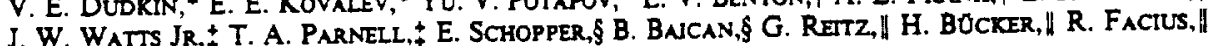 \\ R. Beaujean I and C. HelmanN"
}

- Research Center for Space Flight Radiation Safety, Ministry of Public Health, Moscow 123182, U.S.S.R.; †University of San Francisco, San Francisco, CA 94117, U.S.A.; †NASA-Marshall Spaceflight Center, Huntsville, AL 35812, U.S.A.; §Institute for Nuciear Physics, Frankfurt am Main, Germany; \|DLR Institute for Aerospace Medicine, Köln, Germany; TUniversity of Kiel, Kiel, Germany; " Centre de Recherches Nucléaires, Strasbourg. France

(Received 23 June 1991; in revised form 3 July 1991)

\begin{abstract}
Results of the experiments on board Cosmos-2044 (Biosatellite 9) are presented. Various Abstract-Results of the experiments on board detectors (NTD) (dielectric, AgCl-based, nuclear emulsions) were used to obtain the LET spectra inside and outside the satellite. The spectra from the different NTDs have proved to be in general spectra inside and outside the satelite. The spectra trom the difesults of LET spectra calculations using two different models are also presented. The agreer. quality factors (QF) of the cosmic rays (CR). Absorbed dose rates inside $\left(\sim 20 \mathrm{~g} \mathrm{~cm}^{-2}\right.$ shielding) and outside $\left(\mathrm{g} \mathrm{cm}^{-2}\right)$ the spacecraft, omitting electrons, were found to be 4.8 and $8.6 \mathrm{mad} \mathrm{d}^{-1}$, respectively, while the corresponding equivalent doses were 8.8 and 19.7 mrem $\mathrm{d}^{-1}$. The effects of the llight parameters on the total fluence of, and on the dose from, the CR particles are analyzed. Integral dose distributions of the detected particles are also determined. The LET values which separate absorbed and equivalent doses into $50 \%$ intervals are estimated. The CR-39 dielectric NTD is shown to detect $20-30 \%$ of the absorbed dose and $60-70 \%$ of the equivalent dose in the Cosmos-2044 orbit. The influence of solar activity phase on the magnitude of CR Iux is discussed.
\end{abstract}

\section{INTRODUCTION}

THE RADUTON environment in near-Earth space needs to be shown in order to estimate the radiation risk for any planned manned space mission. The need for this knowledge becomes even more necessary in the case of long-term missions.

Cosmic ray dosimetry is faced with certain difficulties due to the complicated composition of radiation fields in near-Earth space, the diversity of the factors affecting the qualitative and quantitative compositions of the fields, and some characteristic features inherent to space flights. Copious relevant information bas been accumulated within the last decades, including the total absorbed dose, the dose rates, the particle LET spectra, and the particle flux densities. The information relates mainly to lowinclined orbits (inclination $i \leqslant 70^{\circ}$ ) with altitudes not exceeding $\sim 450 \mathrm{~km}$.

Recently, the absorbed and equivalent doses have been estimated using not only dosimeters, but also NTDs which make it possible to measure the integral LET specrra of cosmic ray particles in low orbits. Measurements of that type were made earlier (Benton, 1983, 1986; Benton and Henke, 1983;
Akopova et al., 1985, 1986, 1987, 1988, 1990a) for various satellite orbit parameters and thicknesses of detector shieldings during different solar activity (SA) periods. Some works should also be noted (Benton, 1986; Akopova et al., 1985, 1986, 1987, 1988, 1990a; Heinrich, 1977; Heinrich and Baer, 1984; Heinrich et al., 1989) where the LET distributions were calculated for different satellite parameters. These calculations treated only the galactic cosmic ray (GCR) particle component of the radiation field.

The characteristic features of the Cosmos-2044 flight were as follows: the flight took place during solar maximum and the orbit was near-polar $\left(i=\sim 82^{\circ}\right)$. Various NTDs (dielectric plastics, AgCl single-crystais and nuclear emulsions of different types), mounted inside and outside the satellite, were used to measure the CR paricle LET spectra within a broad range. The present work compares the results of the measurements taken inside and outside (under low shielding thicknesses) the Cosmos-2044 satellite. The resultant LET spectra are used to calculate the total absorbed and equivalent doses and the LET distributions of the doses.

The following four basic factors affect the magnitude of the charged-particle flux measured in

+USF portion of the work partially supported by NASA Grant Nos NCC2-521 (NASA-Ames Research Center) and NAG9-235 (NASA-Johnson Space Center).

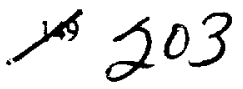


space: (a) solar modulation, i.e. the dependence of paricle flux on SA period; (b) geomagnetic cut-off threshold (the GCR flux increases with satellite orbit inclination); (c) the detector shielding thickness; and (d) the satellite flight altitude (the trapped proton flux increases steeply with orbital altitude). The degree of the effect of these factors on the measured LET distributions will also be discussed below.

Work by Akopova et al. (1990b) and Dudkin et al. (1992) presents the Biosatellite-9 preliminary experimental data including the results obtained by Soviet experts using nuclear emulsions (Akopova el al., 1990b) and the results of the joint Soviet-American researchers (Dudkin et al., 1992). These are compared with the relevant calculated results.

\section{EXPERMMENTAL DESIGN}

In conformity with the scientific program for Biosatellite 9 (Cosmos-2044), the solid-state nuclear track detectors (SSNTDs), i.e. nuclear emulsions, $\mathrm{AgCl}$-based detectors, and dielectric detectors, were placed in specially designed containers and exposed inside and outside the satellite during 14 days of Elight near the Earth (at $294 \mathrm{~km}$ apogee, $216 \mathrm{~km}$ perigee, orbit inclination $i=82.3^{\circ}$ ) under quiet geomagnetic conditions. The participants in the experiment are the ESA, represented by Frankfurt University, Germany (the $\mathrm{AgCl}$ detectors), Kiel University, Germany (the dielectric detectors with different thresholds of particle LET detection), the Centre de Recherches Nucléaires, Strasbourg, France (nuclear emulsions), and the .Research Center of Spacecraft Radiation Safety, Moscow, U.S.S.R. (nuclear emulsions of various types). Plastic nuclear track detectors were contributed by the University of San Francisco, U.S.A. The AgCl detectors were mounted only inside the satellite, and the American-made dielectric detectors only outside the satellite, while the Germanmade dielectric detectors and the French- and Soviet-made nuclear emulsions were mounted both inside and outside the satellite.

The SSNTDs used in the experiment are characterized by effective particle LET thresholds above which the particles could be detected ( $L_{\text {throb }}$ : see Table 1 ).

Various types of nuclear emulsions were used to detect the CR particles throughout their LET range. Emulsion stacks containing 200- $\mu \mathrm{m}$-thick BR- and BYa-type emulsions wrapped with light-tight paper and aluminized Lavsan were placed in instrument modules either outside or inside the satellite. After exposure and recovery, each layer of the emulsion stack was treated by the selective development technique which makes it possible to control the emulsion layer threshold sensitivity in a broad interval of LET (Akopova et al., 1983). The emulsion threshoid sensitivity control is based on the introduction of $\mathrm{Br}^{-}$ions into an exposed emulsion layer by diffusion. The $\mathrm{Br}^{-}$ jons were emitted from BR-type layers (emitters) glued to either of the surfaces of an exposed layer that had been irradiated beforehand with blue-violet light. The $\mathrm{Br}^{-}$ion generation and diffusion from the emitters to the exposed layers gives rise to a negative bromine barrier around the latent image centers, thereby increasing the induction periods of developing centers. The ability of the centers to be developed depends, then, upon the ratio of the height of the barrier restricting the arrival of electrons at the centers to the depth of the potential well in which the electrons are captured. Thus, the emulsion threshold sensitivity control is based on different degrees of dispersion of the latent image centers produced by particles with different LET values. The threshold sensitivity of the exposed layer permits only those particle tracks to be developed for which the LET is at least equal to some threshold LET value. Therefore, the technique of finding the planar fluence of the particles with LET $\geqslant$ LET $_{\text {thres }}$ does not require the track parameters to be measured but is reduced to counting the number of the tracks traversing a particular section of an emulsion surface. Calibration of the technique was achieved by exposing emulsions to particle beams of well-defined LETs.

We used BYa-type emulsion which permits the integral LET spectra to be measured within an interval from 12 to $\sim 10^{4} \mathrm{MeV} \mathrm{cm}^{-1}$ of biological tissue (water), with the lower limit $\left(12 \mathrm{MeV} \mathrm{cm}^{-1}\right)$ being defined by the effective sensitivity of the BYa-type emulsion. To obtain a complete LET distribution, we sometimes used the relativistic BR-type emulsion, thereby making it possible (to within a large microscope scanning error) to find the planar fluence of CR particles at small LET values (the LET of relativistic protons in tissue is $\sim 2.0 \mathrm{MeV} \mathrm{cm}^{-1}$ ). Scanning error with BR-type emulsion increases (a) due to a high track exposure of the detectors (the satellite flights lasted, as a rule, for more than 10 days); and (b) because the operator can easily overlook tracks of relativistic protons (low grain densities).

This method has the drawback of emulsion shrinkage after the layers have been fixed and developed, which may lead to distortion. The necessity for a

Table 1. Effective thresholds $\left(L_{\text {thmin }}\right)$ of particle detection by the Cosmos-2044 NTDs

\begin{tabular}{|c|c|c|c|c|c|c|c|c|}
\hline $\begin{array}{l}\text { Detector } \\
\text { Detector type }\end{array}$ & CR-39 & $\begin{array}{c}\text { Dielectri } \\
\mathrm{CN}_{k}\end{array}$ & $\begin{array}{c}\text { detectors } \\
\mathrm{CN}_{\mathrm{D}}\end{array}$ & Lexan & $\begin{array}{c}\text { Single } \\
\text { crystal } \\
\mathrm{AgCl}\end{array}$ & BR-2 & $\begin{array}{c}\text { Nuclear emulsion } \\
\text { BYa-2 }\end{array}$ & Ilford $\mathrm{K}_{2}$ \\
\hline Country & U.S.A. & Kiel & $\begin{array}{l}\text { Germany } \\
\text { Kiel }\end{array}$ & Kiel & $\begin{array}{l}\text { Germany } \\
\text { Frankfurt }\end{array}$ & U.S.S.R. & U.S.S.R. & France \\
\hline $\begin{array}{l}L_{\text {thrall, }}, \mathrm{MeV} \mathrm{cm}^{-1} \text {, } \\
\text { in tissue }\end{array}$ & $\sim 40$ & 1200 & 1800 & 5800 & 60 & 2.0 & 12.0 & $>10$ \\
\hline
\end{tabular}


$\sim 60 \%$ ambient humidity to be maintained is also a drawback of the method.

The method for finding the LET distributions with dielectric detectors of CR-39, CN, and Lexan types, whose effective thresholds of paricle LET detection are presented in Table I, has been tested earlier during various spacecraft flights. After being etched under appropriate time-temperature conditions, the Kiel detectors were scanned to find the LET spectra by counting the particle track densities per surface and correcting for flight duration and for the effective solid angles for registration of particles incident on the detectors. The particle numbers were then plotted at the respective LET thresholds for each of the detectors.

The American-made CR-39 plastic NTDs, of $\sim 600 \mu \mathrm{m}$ thickness each, were etched in the standard manner for 7 days in a $6.25 \mathrm{~N} \mathrm{NaOH}$ solution at $50^{\circ} \mathrm{C}$. The bulk etch $B$ (i.e. the total thickness removed from each surface) was measured in all the layers. For readout, pairs of detectors were segregated according to their orientations during the flight. Each two adjacent inner surfaces were scanned with an optical microscope, thereby making it possible to group the particles into short-range (SR) tracks, when the matching tracks appear on two inner surfaces only, and the long-range GCR tracks, when the matching tracks appear on all four surfaces of the pairs of detectors. The SR particles included the SR secondaries from target nuclei within the plastic, the stopping primary GCR particles, and the trapped RB particles. All protons were classified as $S R$ particles because of their short registration paths in CR-39. The GCR particles include the primary CR particles and the long-range secondaries which are mainly projectile fragments of primary GCRs. Etching of the CR-39 produces conical tracks at the particle entrance and exit points. The intersections of the cones with the CR-39 surfaces are elliptical in shape. The lengths of the major and minor axes of the ellipses were measured on the detector surfaces. The axial lengths and the values of $B$ in the specimens, together with the calibration of particle LET response in CR-39, were then used to find the total LET distributions.

The $\mathrm{AgCl}$ single-crystal layers coated with a $\mathrm{Cd}$ grease on a glass underlayer were used to obtain the LET spectra inside the satellite. For particle detection by $\mathrm{AgCl}$ crystals, high-power yellow-green light sources are used to preserve the latent track image before development (German-made lithium batteries were used on the satellite). The $140-\mu \mathrm{m}$-thick $\mathrm{AgCl}$ single crystals were mounted inside specially designed containers where biological objects were also placed. Such a detector was used earlier in spaceborme experiments, mainly in radiobiological experiments (for example, on Cosmos-1887). The detectors could record particles with LET above $10 \mathrm{MeV} \mathrm{g}^{-1} \mathrm{~cm}^{-2}$. During the spacellight, the latent image centers produced by the particles with low LET values disappear due to the fading which occurs continually within the detector volume, so the effective detection threshold for particle LET gets shifted towards higher LET values $\left(\sim 60 \mathrm{MeV} \mathrm{cm}^{-1}\right.$ of tissue in the given experiment). The detectors are used to find the LET spectra of CR particles in the LET range above the threshold.

In the present work, the experiments were supplemented with calculations of the LET spectra in the Cosmos-2044 orbit using two independent programs designed in the U.S.A. and in the U.S.S.R. The American program allows for the contributions of GCR particles (protons and He nuclei only), of the inner RB protons, and of the $Z=1$ anomalous component to the integral LET distributions. Allowance is also made for geomagnetic cut-off and for the screening of particles by the Earth without, however, any geomagnetic disturbance. A similar program designed in the U.S.S.R. was used to calculate the contribution from the $Z \geqslant 3$ GCR particles only. More details can be found in Dudkin et al. (1992).

\section{RESULTS AND DISCUSSION}

Figures 1 and 2 show the integral LET spectra outside (Fig. 1) and inside (Fig. 2) Cosmos-2044. The spectra have been obtained with different NTDs. In all cases, the planar particle fluence, i.e. the number of tracks traversing the detector surface, was measured.

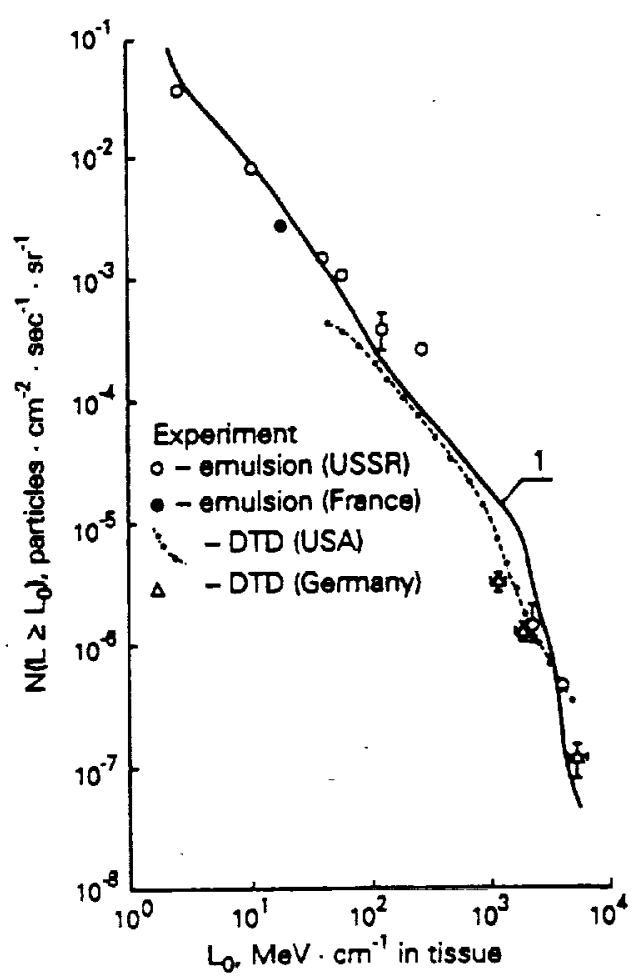

Fic. 1. Comparison between the calculated (curve 1-for Fig. $\mathrm{cm}^{-2}$ ) and experimental integral LET distributions of CR particles obtained on Cosmos-2044 (stacks outside the satellite); DTD denotes plastic dielectric track detectors. 


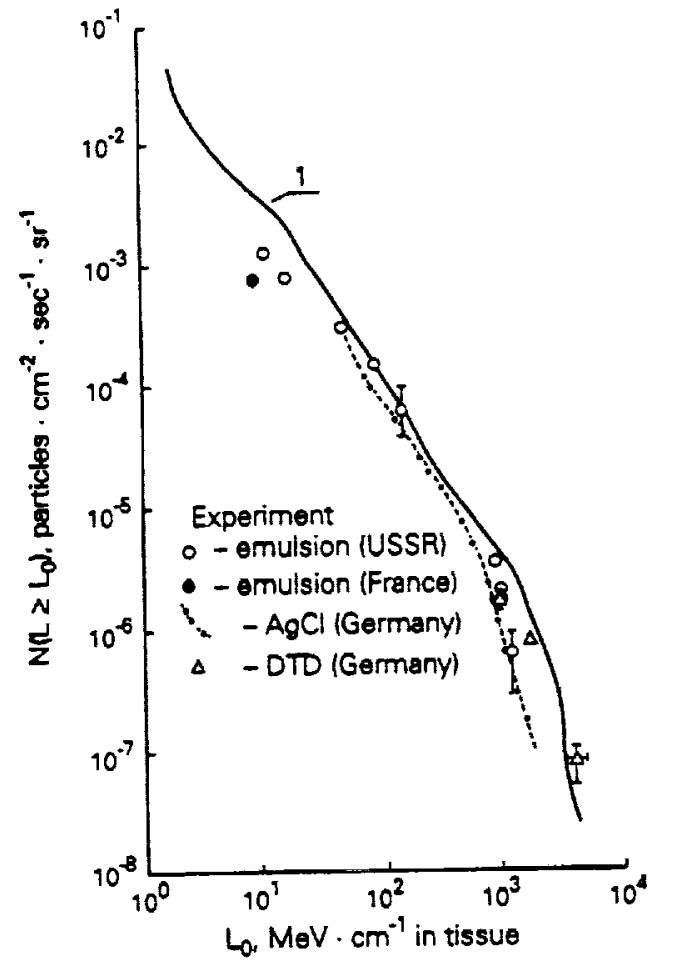

Fig. 2. Comparison between the calculated (curve !-for $20 \mathrm{~g} \mathrm{~cm}^{-2}$ ) and experimental LET distributions of CR particles obtained on Cosmos-2044 (stacks inside the satellite)

DTD denotes plastic dielectric track detectors.

It should be noted that the measurement results obtained at different research centers with different detectors have proved to be in satisfactory agreement. Certain differences in the integral spectra obtained outside the satellite (Fig. 1) can be accounted for by the difference in the detector shielding thicknesses. Because of a near-polar inclination of the satellite orbit, the contribution of SR particles to the total flux must depend substantially on the detector shielding thickness, even in the case of small thicknesses $\left(\delta<2 \mathrm{~g} \mathrm{~cm}^{-2}\right)$. The American-made dielectric detector data show that the SR particle flux density under a shielding $\delta=0.2 \mathrm{~g} \mathrm{~cm}^{-2}$ is almost an order of magnitude higher than that of $\delta \sim 2.0 \mathrm{~g} \mathrm{~cm}^{-2}$ (Dudkin et al., 1992).

For comparison, Figs 1 and 2 also present the calculated integral LET distributions of $C R$ particles on the Cosmos-2044 orbit during solar maximum. The caleulations were made at $\delta=1.0 \mathrm{~g} \mathrm{~cm}^{-2}$ (Fig. 1) and $\delta=20 \mathrm{~g} \mathrm{~cm}^{-2}$ (Fig. 2) using the two available models for calculating the LET spectra. The figures show the total LET spectra of CR particles (curve 1). Each of the distributions consists of an LET spectrum defined by the light $C R$ components (the $G C R$ protons, the He nuclei and the RB protons) as calculated by the American program, and includes the LET distribution of the $Z>3$ CR particles (GCR particles) calculated by the Soviet program. A good agreement is seen between the experimental and theoretical data in the two figures.

It should be noted that the simultaneous usage of different techniques for measuring the LET spectra has proved to be very fruitful because, firstly, a broader range of LET values can be covered and, secondly, the reliability of the measurement results in the intervals of overlapped measurements are improved.

The LET distributions obtained were used to calculate the absorbed and equivalent doses inside and outside the satellite. To facilitate the calculations, the LET spectra were approximated by piecewise continuous functions:

$$
N\left(L \geqslant L_{0}\right)=\alpha \times L_{0}^{-\beta},
$$

where $\alpha$ and $\beta$ are constants.

The integral LET spectra of CR particles measured in different low-Earth orbits and outside the magnetosphere exhibit a characteristic knee at $L \sim 1350$ $\mathrm{MeV} \mathrm{cm}{ }^{-1}$. The knee, i.e. a decrease of the exponent $\beta$ in (1), can be accounted for by the fact that the GCR Fe nuclei are detected at $L \cong 1350 \mathrm{MeV} \mathrm{cm}^{-1}$ and higher, and that the flux of $\mathrm{Fe}$ guclei decreases with increasing $L$ very rapidly in comparison with the range $L<1350 \mathrm{MeV} \mathrm{cm}^{-1}$, where all $\mathrm{CR}$ particles from protons to $F e$ nuclei contribute to the total flux.

The same pattern was recorded on Cosmos-2044; from Figs 1 and 2 it is seen that the characteristic knees in the LET distributions at $L>10^{3} \mathrm{MeV} \mathrm{cm}^{-1}$ are observed. When calculating the doses for these spectra, therefore, we approximated the resultant integral LET spectra by expressions of type (1) in two $L$ ranges, $2.0 \leqslant L_{0} \leqslant 1350 \mathrm{MeV} \mathrm{cm}{ }^{-1}$ and $1350<L_{0}<5 \times 10^{4} \mathrm{MeV} \mathrm{cm}^{-1}$.

To find the effect of the spread of the experimental points in the LET spectra on the absorbed and equivalent doses, we applied the approximation functions of type (1) to each of the spectra shown in Figs 1 and 2 in such a manner that all the experimental and calculated values of $N\left(L \geqslant L_{0}\right)$ would be below (the first case, "Maximum" version) and above (the second case, "Minimum" version) the approximation functions. Table 2 presents the resultant values of the

Table 2. The values of the approximation constants $\alpha$ and $\beta$

\begin{tabular}{llccccc} 
& & \multicolumn{4}{c}{ Detectors mounted } \\
\cline { 3 - 6 } & & \multicolumn{2}{c}{ Outside the satellite } & \multicolumn{2}{c}{ Inside the satellite } \\
\hline Version & LET range, in tissue & constant: & $\alpha$ & $\beta$ & $\alpha$ & $\beta$ \\
Maximum & $2 \leqslant L \leqslant 1350 \mathrm{MeV} \mathrm{cm}^{-1}$ & 0.2088 & 1.3800 & 0.1425 & 1.4540 \\
& $1350<L<5 \times 10^{4} \mathrm{MeV} \mathrm{cm}^{-1}$ & $1.052 \times 10^{3}$ & 2.5626 & $1.00 \times 10^{6}$ & 3.641 \\
Minimum & $2 \leqslant L \leqslant 1350 \mathrm{MeV} \mathrm{cm}^{-1}$ & 0.2370 & 1.5645 & 0.1836 & 1.8197 \\
& $1350<L<5 \times 10^{4} \mathrm{MeV} \mathrm{cm}^{-1}$ & $2.850 \times 10^{6}$ & 3.826 & $5.03 \times 10^{16}$ & 7.39 \\
\hline
\end{tabular}


Table 3. Calculated values of the absorbed $(D)$ and equivalent $(H)$ doses

\begin{tabular}{|c|c|c|c|c|}
\hline \multirow[b]{3}{*}{ Version | Dose } & \multicolumn{4}{|c|}{ Detectors mounted } \\
\hline & \multicolumn{2}{|c|}{$\begin{array}{l}\text { Outside the satellite } \\
\left(1 \mathrm{~g} \mathrm{~cm}^{-2}\right)\end{array}$} & \multicolumn{2}{|c|}{$\begin{array}{l}\text { Inside the satellite } \\
\left(20 \mathrm{~g} \mathrm{~cm}^{-2}\right)\end{array}$} \\
\hline & $D, \frac{\mathrm{mrad}}{\mathrm{day}}$ & $H, \frac{\text { mrem }}{\text { day }}$ & $D, \frac{\text { mrad }}{\text { day }}$ & $H, \frac{\text { mrem }}{\text { day }}$ \\
\hline $\begin{array}{l}\text { Maximum } \\
\text { Minimum } \\
\text { Mean doses }\end{array}$ & $\begin{array}{c}9.7 \\
7.6 \\
8.6 \pm 1.4\end{array}$ & $\begin{array}{c}25.9 \\
13.6 \\
19.7 \pm 6.2\end{array}$ & $\begin{array}{c}5.6 \\
4.0 \\
4.8 \pm 0.8\end{array}$ & $\begin{array}{c}12.5 \\
5.1 \\
8.8 \pm 3.7\end{array}$ \\
\hline
\end{tabular}

constants $\alpha$ and $\beta$. Table 3 presents the doses calculated throughout the $L$ range in two versions and also gives the mean values of the doses.

The QFs averaged over the LET spectra on the given orbit are 2.3 outside and 1.8 inside the satellite. It should be noted that the values of the doses and QFs obtained outside the satellite do not allow for the contribution to the total dose from the RB electrons which, according to Akatov et al. (1990), may constitute $70-90 \%$ of the total dose under shielding of less than $\sim 1.5 \mathrm{~g} \mathrm{~cm}^{-2}$ (even during solar minimum and at an orbital inclination of $60^{\circ}$ ). With the electron component included, the doses during solar maximum at $82^{\circ}$ inclination and $250 \mathrm{~km}$ altitude will increase to $D(\delta=1.0)=69.3 \mathrm{mrad}^{-1}$ and $H(\delta=1.0)=83.6 \mathrm{mrem} \mathrm{day}^{-1}$. The averaged QF on such an orbit under shielding $\delta=1.0 \mathrm{~g} \mathrm{~cm}^{-2}$ will fall to $\mathrm{QF}=1.2$, in agreement with Akatov et al. (1990).

The equivalent doses were calculated using our approximation of the dependences of $Q F$ on LET $\equiv L_{\infty}$ in tissue, the values of which are tabulated in ICRP-26 (1977):

$$
\left.\begin{array}{l}
\text { QF }=1 \\
\text { at } L \leqslant 35 \mathrm{MeV} \mathrm{cm}^{-1} \\
\text { QF }=2.857 \times 10^{-2} \times L \\
\text { at } 3.5<L \leqslant 70 \mathrm{MeV} \mathrm{cm}^{-1} \\
\text { QF }=6.830 \times 10^{-2} \times L^{0.7950} \\
\text { at } 70<L \leqslant 530 \mathrm{MeV} \mathrm{cm}^{-1} \\
\text { QF }=2.625 \times 10^{-1} \times L^{0.5803} \\
\text { at } 530<L \leqslant 1750 \mathrm{MeV} \mathrm{cm}^{-1} \\
\text { QF }=20 \\
\text { at } L>1750 \mathrm{MeV} \mathrm{cm}^{-1} .
\end{array}\right\}
$$

The disagreement between the approximated data and the recommended QF values is not greater than $\pm 5 \%$ throughout the $L$ range. It should also be noted that the CR doses were calculated in some studies (Silberberg et al., 1984; Letaw et al., 1986, 1987; Letaw and Clearwater, 1986) using otber functions to approximate the dependence $\mathrm{QF}=f(L)$ :

$$
\mathrm{QF}= \begin{cases}1 & \text { at } L \leqslant 35 \mathrm{MeV} \mathrm{cm}^{-1} \\ 0.072 \times L^{0.74} & \text { at } 35<L<2000 \mathrm{MeV} \mathrm{cm}^{-1} \\ 20 & \text { at } L \geqslant 2000 \mathrm{MeV} \mathrm{cm}^{-1}\end{cases}
$$

An analysis has shown that the equations (3) underestimate the recommended $Q F$ values by up to $20-30 \%$ at $35<L<2000 \mathrm{MeV} \mathrm{cm} \mathrm{cm}^{-1}$. It is noteworthy that the absorbed doses calculated in the versions "Maximum" and "Minimum" (see Table 3) differ from each other by not more than $\sim 30 \%$ for the LET spectra measured both inside and outside the satellite. At the same time, the equivalent doses differ from each other by a factor of 2.0-2.5 for the same LET distributions. It should be emphasized that this corresponds to the largest spreads found between the different measurements.

Figure 3 presents our calculated integral LET distributions of the absorbed and equivalent doses outside and inside the satellite. The approximation functions in the version "Maximum" were used as the input data. From Fig. 3 it is seen that the general trend of the LET dependence is the same outside and inside the satellite with the dependence being "shifted" towards smaller LET values (towards higher particle energies) inside the satellite, as would be expected in principle.

Nearly $50 \%$ of the total equivalent dose is from the particles whose LET exceeds $400 \mathrm{MeV} \mathrm{cm}^{-1}$, whereas $\sim 50 \%$ of the absorbed dose is from the particles with LET below $10 \mathrm{MeV} \mathrm{cm}^{-1}$. Knowing, for example, that dielectric detectors such as CR-39 measure particles with LET $\geqslant 40 \mathrm{MeV} \mathrm{cm}^{-1}$ in tissue, we can note from Fig. 3 that, in this instance, the detected

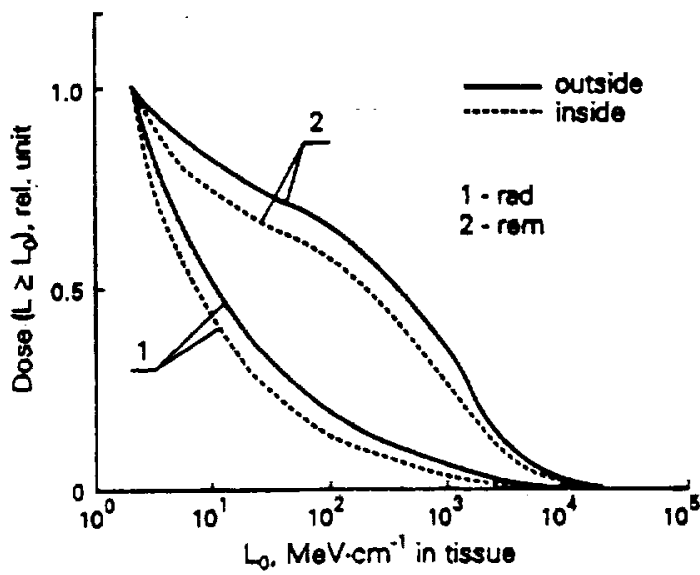

FIg. 3. The dose-LET distributions obtained on Cosmos2044. The outside curves are for $1 \mathrm{~g} \mathrm{~cm}^{-2}$ shielding; inside curves are for $20 \mathrm{~g} \mathrm{~cm}^{-2}$. 
absorbed dose can be as small as 20-30\% of the total, while the equivalent dose is $60-70 \%$ of the total. This fact has been corroborated by the data of Dudkin el al. (1992) where absorbed and equivalent doses which were measured with CR-39 outside Cosmos2044 are presented. According to Fig. 3, the absorbed dose $\left(1.32-1.53 \mathrm{mrad}\right.$ day $\left.{ }^{-1}\right)$ under an $\sim 1.5 \mathrm{~g} \mathrm{~cm}^{-2}$ shielding is $23 \%$ of the total dose, thereby yielding $\sim 6.2 \mathrm{mrad} \mathrm{day}^{-1}$ when the total dose is estimated for the full LET range, in agreement with the mean value of $(8.6 \pm 1.4) \mathrm{mrad}^{-1} \mathrm{yy}^{-1}$ from Table 3 at $\sim 1.0 \mathrm{~g} \mathrm{~cm}^{-2}$. Similarly, according to Fig. 3 , the value of (12.8-13.9) mrem day ${ }^{-1}$ must constitute $\sim 72 \%$ of the total dose. Simple calculations yield $18.5 \mathrm{mrem}$ day $y^{-1}$, also in good agreement with the data of Table 3 for outside the satellite.

The greatest differences in the values of the LET spectra measured by different rechniques occur in the tails of the spectra. The difierences may be accounted for by a low statistical accuracy of the results for rare events (particles with high LET values) and by marginal detection of the fluence of CR paricles with LETs near the detector thresholds. Particular attention should be paid in the future to the correct determination of the total fluence (at the lowest ionization values) and of the fluence at $L>10^{3} \mathrm{MeV} \mathrm{cm}^{-1}$.

- As noted above, the Cosmos-2044 flight was distinguished by the following factors: the fight took place during solar maximum on a near-polar orbit with an apogee of close to $300 \mathrm{~km}$. The effect of the orbital parameters on the different sources of radiation in the Earth's environment (GCR particles and RB protons and electrons) varies. The GCR particle fux changes little with orbital altitude, but decreases with increasing solar activity (due to the screening effect of solar wind on the GCR flux) and increases with orbital inclination (due to a decrease in the geomagnetic cut-off threshold). The RB proton contribution decreases with increasing solar activity and with decreasing orbital altitude (at $\sim 200-300 \mathrm{~km}$ the orbit passes under the zone of the most intense fluxes of the Brazilian anomaly) and increases with decreasing $i$, for $i>\sim 35^{\circ}$ (due to a longer residence of the satellite in the region of the Brazilian anomaly). The RB electron fluxes increase with solar activity, whereas the contribution of precipitating electrons in the Earth's near-polar zone increases with decreasing altitude and increasing inclination.

The above-mentioned relationships bave been confirmed experimentally by comparing doses measured with American-made thermoluminesceat dosimeters on two satellites (Cosmos-2044 and -1887) (Frank et al., 1990; Benton et al., 1988). Cosmos-1887 flew at a bigher altutude and a smaller inclination during solar minimum.

As noted above. the Cosmos-2044 flight is distinguished by its coincidence with the maximum of Solar Cycle 22. According to some predictions [see the review ISSN 0208-1849 (1990)] the Cycie 22

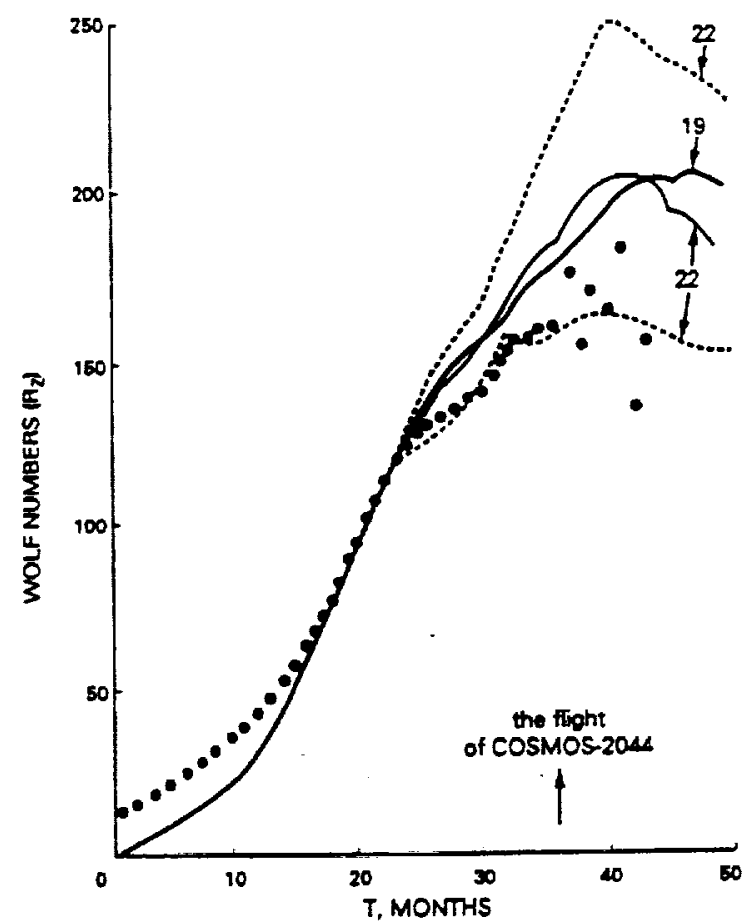

Fig. 4. Wolf numbers versus time during Solas Cycle 22.

maximum should have exceeded all the previous cycles in solar activity. If so, we would expect a pronounced decrease in the GCR particle flux and in the RB proton flux in low orbits. This was not observed, however.

The Wolf numbers $\left(R_{z}\right)$, recorded continually at various ground-based observatories, are known to be a significant criterion for estimating solar activity. Figure 4, borrowed from ISSN 0208-1849 (1990), presents the Wolf number data up to March 1990. September 1986 is taken to be the onset of Cycie 22; the Cycle 19 data are presented for comparison (Cycle 19 was the most intense of the last five decades). The thick solid line represents the $R_{7}$ values during Cycle 19 (smoothed mean-monthly $R_{\text {. }}$ values); the dashed lines represent a $90 \%$ confidence interval; the dots are observation data (smoothed $R$, values up to August 1989 and observed values up to March 1990).

From Fig. 4 it is seen that, up to the present, a maximum of extreme intensity has not been attained in Cycle 22. At most, the $R_{a}$ values will approach the values observed during Cycle 19, but will not exceed them. Thus, despite the violent rise of solar activity during its initial phases, Cycle 22 can be considered to be fairly moderate, with the highest values of $R_{z}$ reaching 150-170, which are close to the levels during Cycles 18 and 19.

Considering the fact that Cosmos-2044 fiew in September 1989 and that the delay between solar activity and GCR particle modulation is about half a year, we may say that solar activity during the Cosmos-2044 tight was moderate and comparable with the levels during the previous solar cycle. Our results are consistent with this conclusion. 


\section{CONCLUSIONS}

(i) The experimental studies of integral LET distributions carried out on board Cosmos-2044 by experts from different countries using various detectors have demonstrated that the results obtained are in satisfactory agreement in the overlapping LET ranges. A satisfactory agreement was also obtained between the experimental data and the LET spectrum calculations obtained with two different calculation models.

(ii) The absorbed and equivalent doses inside and outside the satellite have been calculated using the measured LET distributions as input data. This approach has been demonstrated to yield good results for calculating the absorbed dose and to be applicable for estimating the equivalent dose. For improved results, it is necessary to increase the accuracy of flux measurements in the tails of the LET spectra and, particularly, to measure the fluence of all charged heavy $C R$ particles by multiple methods.

(iii) The integral dose LET distributions calculated for Cosmos-2044 show that $\sim 50 \%$ of the absorbed dose is from the particles with LET below $10 \mathrm{MeV}$ $\mathrm{cm}^{-1}$, while $\sim 50 \%$ of the equivalent dose is from the particles with LET above $400 \mathrm{MeV} \mathrm{cm}{ }^{-1}$. The CR.39 detector, with a LET threshold for paricle detection at $\sim 40 \mathrm{MeV} \mathrm{cm}^{-1}$, may detect as little as $20-30 \%$ of the absorbed dose and $60-70 \%$ of the equivalent dose in a Cosmos-2044 type of orbit.

(iv) The Cosmos-2044 fight is distinguished by a near-polar orbit and by its coincidence with solar maximum. The orbit tends to increase the detected particle fluence, in comparison with the usual lower inclination orbits, due to a decrease in the geomagnetic cutoff threshold. The coincidence with solar maximum, on the other hand, results in a general decrease in fluences of both GCR particles and RB protons. These factors, along with a rather low orbital altitude, explain the variations in radiation exposure as compared with earlier spaceflights. It has also been demonstrated that, despite the predictions of an extremely high solar maximum in Cycie 22, the solar acrivity can be considered fairly moderate. This is consistent with the experimental data presented bere.

\section{REFERENCES}

Akatov Yu. A., Dudkin V. E., Kovalev E. E., Benton E. V., Frank A. L. Warts J. W. Jr and Pamell T. A. (1990) Depth distribution of absorbed dose on the extemal surface of Cosmos-1887 biosatelilite. Nucl. Traciks Radiat. Meas. 17, 105-107.

Akopova A. B., Dudkio V. E., Karpov O. N., Melkumyan L. V., Potapov Yu. V. and Rshruni Sh. B. (1986) Determination of cosmic radiation characteristics aboard Salyut-7 orbital station. Nuel. Tracks Radiat. Meas. 12, 489-491.

Akopova A. B., Dudkin V. E., Karpov O. N.. Melkumyan L. V., Polapov Yu. V. and Rshruni Sh. B. (1988) Determination of cosmic radiation characteristics on board Salyut-7 orbital station. Kosm. Issled. XXVI, $162-165$.
Akopova A. B., Dudkin V. E., Kovalev E. E., Magradze N. V. and Potapov Yu. V. (1987) Linear energy uransfer spectra of cosmic radiation aboard Cosmos. 1129 arificial satellite. Radiat. Prot. Dosim. 18, 153-156.

Akopova A. B., Magradze N. V., Dudkin V. E., Kovalev E. E., Potapov Yu. V., Benton E. V., Frank A. L., Benton E. R., Parnell T. A. and Watts J. W. Jr (1990a) Linear energy transfer (LET) spectra of cosmic radiation in low Earth orbit. Nucl. Tracks Radiat. Meas. $17,93-97$.

Akopova A. B., Magradze N. V., Moisenko A. A., Muradyan S. H. and Ovnanyan K. M. (1983) Method of selective development of thick-layer nuclear emulsions. Preprint EFI-671 (61)-83, Yerevan Physical Institute, Yerevan, U.S.S.R.

Akopova A. B., Vikhrov A. I., Dudkin V. E., Magradze N. V., Moiseenko A. A., Muradyan A. H., Ovnanyan K. $\ddot{M}$. and Potapov Yu. V. (1985) Measuring the linear energy transfer spectra of cosmic radiation aboard the Cosmos-1129 satellite. Kosm. Issled. XXII, $479-481$

Akopova A. B., el al. (1990b) Determination of radiation environments on board Cosmos-2044 by the nuclear emulsion method. XVth Conf. Nucl. Track Delectors, Marburg, Germany, 3-15 September.

Benton E. V. (1983) Dosimetric radiation measurements in space. Nucl. Tracks Radiat. Meas. 7, 1-11.

Benton E. V. (1986) Summary of radiation dosimetry results on U.S. and Soviet manded spacecraft. Adb. Space Res 6(11), 315-328.

Benton E. V., Frank A. L., Benton E. R., Dudkin V. E. and Marenny A. M. (1988) Radiation experiments on Cosmos 1887: K-6-24, K-6-25, K-6-26. University of San Francisco Report USF-TR-74.

Benton E. V. and Henke R. P. (1983) Radiation exposures during space flight and their measurements. Adv. Spoce Res. 3, 171-185.

Dudkin V. E., Karpov O. N., Potapov Yu. V., Akopova A. B., Magradze N. V., Moiseenko A. A., Benton A. V., Frank A. I. and Watts J. W. Jr (1992) Experimental and calculated LET distributions in the Cosmos 2044 biosatellite orbit. Nucl. Tracks Radias. Meas. 20, 143-147.

Frank A. L., Benton E. V., Benton E. R, Dudkin V. E. and Marenny A. M. (1990) Radiation experiments on Cosmos 2044: K-7-41, Pars A, B, C, D, E. University of San Francisco Report USF.TR-76.

Heintich W. (1977) Calculation of LET spectra of heavy cosmic ray nuclei at various absorbed depths. Radiat. Effects 34, 143-148.

Heinrich W. and Baer J. (1984) The radiation situation in space and its modification by geomagnetic field and shieldings. Adu. Space Res. 4, 133-143.

Heinrich W., el al. (1989) LET spectra of cosmic-ray nuclei for near-Earth orbits. Radiat. Res. 118, 63-82.

ICRP.26 (1977) Recommendations of the International Commission on Radiological Prolection. ICRP Publications, Pergamon Press, U.K.

ISSN 0208-1849 (1990) Aerospace Technologies, No. 8. August. Mir, Moscow.

Letaw J. R. and Clearwater S. (1986) Radiation shielding requirements on long-duration space mission. SCC Report 86-02, July.

Letaw J. R., Silberberg R. S. and Tsao C. H. (1986) Natural radiation hazards on the manned Mars mission. NASA Reporr M002, pp. 642-655, June.

Letaw J. R.. Silberberg R. S. and Tsao C. H. (1987) Radiation hazards on space missions. Nature 330 , $709-710$

Silberberg R. S., Tsao C. H.. Adams J. H. Jt and Letaw J. R. (1984) Radiation doses and IET distributions of cosmic rays. Radiat. Res. 98, $209-226$.

$20\}$ 
